\title{
CENTERED CONVEX BODIES AND INEQUALITIES FOR CROSS-SECTION MEASURES
}

\author{
Horst MARTINi AND ZoKhrab MustafaeV
}

Abstract. The purpose of this paper is to establish some new results on cross-section measures of centered convex bodies. More precisely, we show some connections between inequalities referring to cross-section measures and well-known affine isoperimetric inequalities. Based on this, we derive affine inequalities involving also new characterizations of ellipsoids. In addition, related results on three-dimensional zonoids are obtained. Some of our results are also interesting from the viewpoint of the geometry of finite dimensional real Banach spaces.

Mathematics subject classification (2010): 46B20, 52A20, 52A21, 52A40.

Keywords and phrases: Affine isoperimetric inequalities, Busemann's intersection inequality, crosssection measures, dual mixed volumes, ellipsoids, intersection body, Minkowski geometry, mixed volumes, Petty's projection inequality, projection body, zonoids.

\section{REFERENCES}

[1] N. S. BRAnnen, Volumes of projection bodies, Mathematika 43 (1996), 255-264.

[2] R. J. Gardner, Geometric Tomography, second edition, Encyclopedia of Mathematics and its Applications 58, Cambridge University Press, New York, 2006.

[3] P. Goodey, And W. Weil, Zonoinds and generalisations, In: Handbook of Convex Geometry, Vol. B, pp. 1297-1326, North-Holland, Amsterdam, 1993.

[4] E. Lutwak, Selected affine isoperimetric inequalities, In: Handbook of Convex Geometry, Eds. P. M. Gruber and J. M. Wills, Vol. A, pp. 151-176, North-Holland, Amsterdam et al., 1993.

[5] E. Lutwak, On a conjectured projection inequality of Petty, Contemp. Math. 113 (1990), 171-182.

[6] E. JR. MAKAI, AND H. MARTINI, The cross-section body, plane sections of convex bodies and approximation of convex bodies I, Geom. Dedicata 63 (1996), 267-296.

[7] H. MARTini, Extremal equalities for cross-sectional measures of convex bodies, In: Proc. 3rd Congress of Geometry (Thessaloniki, 1991), Aristotle Univ. Press, Thessaloniki, 1992, 285-296.

[8] H. Martini, and Z. Mustafaev, Some applications of cross-section measures in Minkowski spaces, Period. Math. Hungar. 53 (2006), no. 1-2, 185-197.

[9] H. Martini, And Z. Mustafaev, On unit balls and isoperimetrices in normed spaces, Colloq. Math. 127 (2012), 133-142.

[10] C. M. PetTy, Isoperimetric problems, In Proceedings of the Conference on Convexity and Combinatoral Geometry (Norman, OK, 1971), Dept. Math., Univ. Oklahoma (Norman, OK, 1971), 26-41.

[11] C. A. Rogers, AND G. C. Shephard, Convex bodies associated with a given convex body, J. London Math. Soc. 33 (1958), 270-281.

[12] C. Saroglou, Volumes of projection bodies of some classes of convex bodies, Mathematika 57 (2011), 329-353.

[13] R. SchneIder, Convex Bodies: The Brunn-Minkowski Theory, Encyclopedia of Mathematics and its Applications 44, Cambridge University Press, 1993.

[14] J. E. Spingarn, An inequality for sections and projections of a convex set, Proc. Amer. Math. Soc. 118 (1993), 1219-1224.

[15] A. C. Thompson, Minkowski Geometry, Encyclopedia of Mathematics and its Applications 63, Cambridge University Press, 1996. 
[16] R. Schneider, AND W. Weil, Zonoids and related topics, In: Convexity and its Applications, pp. 296-317, Birkhäuser, Basel, 1983. 